\title{
Gene polymorphisms and expression levels of interleukin-6 and interleukin-10 in lumbar disc disease: a meta-analysis and immunohistochemical study
}

\author{
Yewen Guan ${ }^{1}$, Siting Wang ${ }^{1}$, Jiaqi Wang ${ }^{1}$, Dihua Meng ${ }^{1}$, Huihong Wu', Qingjun Wei ${ }^{2^{*}}$ and Hua Jiang ${ }^{1,2^{*}}$ (1)
}

\begin{abstract}
Background: To investigate the association between interleukin-6 (IL-6) (rs1800795, rs1800796, rs1800797, rs13306435, rs2069849) and interleukin-10 (IL-10) (rs1800871, rs1800896) gene polymorphisms, expression levels, and lumbar disc disease (LDD).

Methods: We conducted a literature research on PubMed, Embase, Web of Science, Cochrane Library, and China National Knowledge Infrastructure (CNKI) until February 28, 2019. We included all case-control studies about the association between IL-6 and IL-10 gene polymorphisms and LDD. The odds ratio (OR) and 95\% confidence interval (Cl) were calculated to estimate the strength of association. Statistical analysis was conducted by Review Manager (RevMan) 5.3 software. Furthermore, immunohistochemistry (IHC) and RT-PCR were performed to evaluate IL-6 and $\mathrm{IL}-10$ expressions in the normal and degenerated disc.

Results: A total of 6 studies, involving 1456 cases and 1611 controls, were included in this meta-analysis. G alleles of rs1800795 and rs1800797 in the IL-6 gene were significantly associated with LDD (rs1800795: G vs. C, OR=1.38, 95\% Cl=1.16-1.64, $P=0.0002 ;$ rs1800797: G vs. A, $O R=1.35,95 \% C l=1.14-1.61, P=0.0006)$. Begg's funnel plot and Egger's tests did not show any evidence of publication bias. IL-6 expression and IL-6 mRNA levels were significantly increased in the degenerated disc compared with those in the normal disc (IL-6 immunopositive cells, $73.68 \pm$ $10.99 \%$ vs. $37.23 \pm 6.42 \%, P<0.001)$.

Conclusions: IL-6 gene polymorphisms (rs1800795 and rs1800797) were significantly associated with susceptibility to LDD. A high expression level of IL-6 may be an important risk factor for LDD.
\end{abstract}

Keywords: IL-6, IL-10, Lumbar disc disease, Meta-analysis, Polymorphisms

\section{Introduction}

Low back pain (LBP) is a common musculoskeletal disorder affecting the general and athletic populations. Over $80 \%$ of the people will suffer from LBP at least one episode in their lifetime [1]. LBP is associated with significant disability and a reduction in quality of life, thus posing a high burden to society $[2,3]$. Lumbar disc degeneration (LDD) is one of the major causes of LBP [4].

\footnotetext{
* Correspondence: gxspine@163.com; drjianghua@163.com

${ }^{2}$ Department of Orthopaedic Surgery, The First Affiliated Hospital of Guangxi Medical University, No.6 Shuangyong Road, Nanning 530021, China

${ }^{1}$ Division of Spine Surgery, The First Affiliated Hospital of Guangxi Medical University, No.6 Shuangyong Road, Nanning 530021, China
}

The etiology of LDD is unclear, but various genetic and environmental factors have been identified [5-7]. Recently, increasing evidence suggested that several genes, such as interleukins (ILs), collagen IX, and matrix metalloproteinases (MMPs), play a vital role in the initiation and progression of LDD $[8,9]$.

Over the past few years, the associations of IL- 6 and IL10 gene polymorphisms with LDD have been investigated in a multitude of studies [10-15]. In 2005, NoponenHietala et al. [10] first studied the association between IL6 and IL-10 gene polymorphisms (rs1800795, rs1800796, rs1800797, rs13306435, rs2069849, and rs1800896) and LDD risk. The result showed that rs13306435 of IL-6 gene

(c) The Author(s). 2020 Open Access This article is distributed under the terms of the Creative Commons Attribution 4.0 International License (http://creativecommons.org/licenses/by/4.0/), which permits unrestricted use, distribution, and reproduction in any medium, provided you give appropriate credit to the original author(s) and the source, provide a link to the Creative Commons license, and indicate if changes were made. The Creative Commons Public Domain Dedication waiver (http://creativecommons.org/publicdomain/zero/1.0/) applies to the data made available in this article, unless otherwise stated. 
was associated with LDD in the Finnish population. Subsequently, other studies contradicted the previous study stating that rs13306435 was not relevant to LDD, but other SNPs (rs1800795, rs1800796, and rs1800797) in the IL-6 gene were relevant to LDD [11, 12, 14, 15]. Moreover, several studies demonstrated that IL-10 gene polymorphisms (rs1800871, rs1800896) were associated with LDD susceptibility $[13,15]$. However, the results were not confirmed by the following studies [14]. Despite extensive research in this field, the results were generally inconsistent and inconclusive. In view of these considerations, we undertook a meta-analysis to investigate the association between IL- 6 and IL-10 gene polymorphisms and LDD. The SNPs rs1800795, rs1800796, and rs1800797 were located in the IL-6 gene promoter, and rs1800871 and rs 1800896 were in the promoter area of the IL-10 gene. These functional SNPs could increase the transcriptional activity of IL- 6 and IL-10 promoter, leading to the upregulation of IL-6 and IL-10 in stress or infection $[16,17]$. In addition, polymorphisms (rs13306435 and rs2069849) in the IL- 6 gene exon have been reported to be associated with different profiles of plasma IL-6 response to immunization [18]. As polymorphisms of IL- 6 and IL-10 gene may alter IL-6 and IL-10 expressions [19], we used immunohistochemistry (IHC) and RT-PCR to evaluate IL6 and IL-10 expression levels in intervertebral disc between the LDD patients and the control subjects.

\section{Materials and methods}

\section{Strategy for literature search}

This meta-analysis was conducted in accordance with a prespecified protocol registered with PROSPERO International Prospective Register of Systematic Reviews (CRD42019124118). Two authors (YW Guan and ST Wang) searched all major databases up to February 28, 2019, including PubMed, Embase, Web of Science, Cochrane Library, and China National Knowledge Infrastructure (CNKI). The following keywords were used for searching: ("LDD" OR "lumbar disc disease" OR "lumbar disc degeneration" OR "intervertebral disc degeneration") AND ("IL-6" or "interleukin-6" OR "IL-10" OR "interleukin-10") AND ("SNP" OR "polymorphisms"). In addition, we checked the reference lists of the included studies for further relevant literature. No publication date or language restrictions were implemented. The authors strictly adhered to the Preferred Reporting Items for Systematic Review and Meta-Analysis Protocols (PRISMA-P) guidelines [20] throughout the study.

\section{Inclusion and exclusion criteria}

To include in all the eligible articles and exclude ineligible articles, the inclusion and exclusion criteria were established. The studies were included in the review if they (1) recruited the experimental subjects which were diagnosed as LDD by clinical or/and radiological examination, (2) were cohort-based or case-control studies that assessed the associations of IL- 6 and IL-10 gene polymorphisms with LDD, (3) evaluated the genotype of the control group and conformed to the HardyWeinberg balance, and (4) calculated the odds ratios (OR) to assess the association. Exclusion criteria were (1) repeated publications; (2) conference abstracts, letters to editor, and unpublished studies; and (3) incomplete data on allele and genotype frequencies. Articles published in languages other than English were translated. Based on the inclusion and exclusion criteria, two authors (YW Guan and ST Wang) independently screened the titles and abstracts of references and obtained the full text for reference. Disagreements between two authors were resolved by discussion, and if necessary, a third author (H Jiang) was consulted.

\section{Data extraction}

Based on a standardized form, two authors (YW Guan and ST Wang) independently extracted data on outcomes for each study. For disagreements, a consensus was reached by a third author ( $\mathrm{H}$ Jiang). Extracted parameters included the following: (1) first author, (2) publication year, (3) study population (country, ethnicity), (4) study design, (5) numbers of cases and controls, (6) characteristics of participants (age and gender), (7) diagnostic criteria, (8) source of controls, (9) allele or/and genotype frequencies, and (10) results of HardyWeinberg equilibrium (HWE) test. Hardy-Weinberg equilibrium was checked in study controls using the $\chi^{2}$ goodness-of-fit test as a quantitative assessment for potential selection bias and confounding.

\section{Methodological quality}

Methodological quality of studies was independently assessed by two investigators according to a quality evaluation form (Critical Appraisal Skills Programme for case- control study, CASP), which is based on ten questions associated with information given by each article [21]. Each question has three degrees behind, "yes" (scored 2), "cannot tell" (scored 1), and "no" (scored 0). The maximum total score is 20 . Studies could be divided into three grades: grade A (high quality, scored 15-20), grade B (medium quality, scored 8-14), and grade C (low quality, scored 0-7).

\section{Study population}

Based on our previous study [22], we collected degenerative disc tissues $(n=34)$ and normal disc tissues $(n=21)$ from the LDD patients and the control subjects. LDD patients were diagnosed with lumbar disc herniation by physical examination and MRI scan. The control subjects were the patients with traumatic lumbar vertebral 


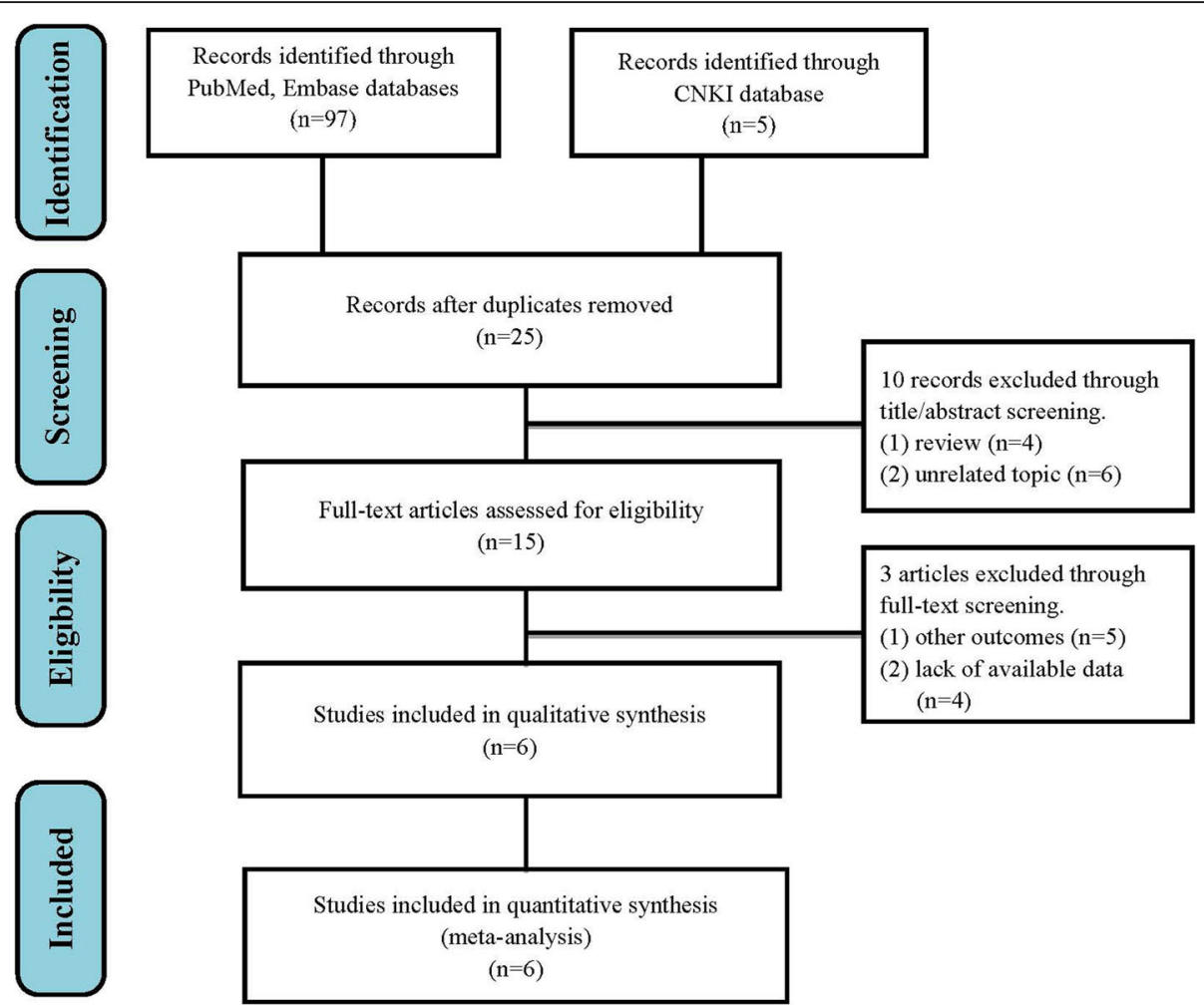

Fig. 1 Literature search strategy and selection of articles. A total of 102 articles were selected for the meta-analysis by browsing the databases PubMed, Embase, and CNKI, of which 77 articles were excluded after reviewing the title and abstract and 10 articles were excluded after reviewing the full publications. Finally, a total of 6 studies were considered for the meta-analysis

fracture, who had no history of low back pain. Based on Schneiderman's classification [23], MRI evaluation showed no significant disc damage and degeneration before surgery (Schneiderman's classification, grade 1, 19 cases; grade 2, 2 cases). These disc samples were used to evaluate IL-6 and IL-10 expressions via IHC and RTPCR. This study was approved by the institute's ethics committee for human studies (2018-KY-NSFC-025). Informed consent was obtained from all the participants in this study.

Table 1 Characteristics of the case-control studies included in meta-analysis

\begin{tabular}{|c|c|c|c|c|c|c|c|c|c|c|c|}
\hline \multirow[t]{2}{*}{ First author } & \multirow[t]{2}{*}{ Year } & \multirow[t]{2}{*}{ Country } & \multirow[t]{2}{*}{ Ethnicity } & \multicolumn{2}{|c|}{$\begin{array}{l}\text { Age (year, } \\
\text { mean } \pm \text { sd) }\end{array}$} & \multirow{2}{*}{$\begin{array}{l}\text { Number } \\
\text { cases/ } \\
\text { controls }\end{array}$} & \multirow[t]{2}{*}{$\begin{array}{l}\text { Diagnostic } \\
\text { criteria }\end{array}$} & \multirow[t]{2}{*}{ Control group } & \multirow[t]{2}{*}{$\begin{array}{l}\text { Outcome measure (SNP } \\
\text { genotyping) }\end{array}$} & \multirow{2}{*}{$\begin{array}{l}\text { HWE } \\
\text { for } \\
\text { controls }\end{array}$} & \multirow[t]{2}{*}{ CASP } \\
\hline & & & & Cases & Controls & & & & & & \\
\hline $\begin{array}{l}\text { Noponen- } \\
\text { Hietala [10] }\end{array}$ & 2005 & Finland & Caucasian & $44 \pm 13$ & $39 \pm 13$ & $155 / 179$ & $M R I$ & NM & $\begin{array}{l}\text { rs1800795, rs1800796, } \\
\text { rs1800797, rs2069849, } \\
\text { rs1800896 }\end{array}$ & 0.97 & 16 \\
\hline Eskola [11] & 2010 & Denmark & Caucasian & $\begin{array}{l}13.1 \pm \\
0.4\end{array}$ & $\begin{array}{l}13.1 \pm \\
0.4\end{array}$ & $66 / 154$ & $M R I$ & $\begin{array}{l}\text { Patients without } \\
\text { LDD }\end{array}$ & $\begin{array}{l}\text { rs1800795, rs1800796, } \\
\text { rs1800797, rs2069849, } \\
\text { rs13306435 }\end{array}$ & 0.74 & 17 \\
\hline $\begin{array}{l}\text { Kelempisioti } \\
{[12]}\end{array}$ & 2011 & Finland & Caucasian & 19 & 19 & $150 / 246$ & MRI & $\begin{array}{l}\text { Patients without } \\
\text { LDD }\end{array}$ & $\begin{array}{l}\text { rs1800795, rs1800796, } \\
\text { rs1800797, rs13306435 }\end{array}$ & 0.92 & 17 \\
\hline $\operatorname{Lin}[13]$ & 2011 & China & Asian & $\begin{array}{l}46.3 \pm \\
8.4\end{array}$ & $\begin{array}{l}46.5 \pm \\
9.7\end{array}$ & $320 / 269$ & $C T, M R I$ & NM & rs1800896, rs1800871 & 0.28 & 19 \\
\hline Huang [14] & 2017 & China & Asian & $\begin{array}{l}44.05 \pm \\
9.02\end{array}$ & $\begin{array}{l}41.85 \pm \\
11.02\end{array}$ & $267 / 300$ & MRI & $\begin{array}{l}\text { Patients without } \\
\text { spine-related } \\
\text { problems }\end{array}$ & $\begin{array}{l}\text { rs1800795, rs1800796, } \\
\text { rs1800896, rs1800871 }\end{array}$ & 0.93 & 18 \\
\hline Zhu [15] & 2017 & China & Asian & $\begin{array}{l}50.27 \pm \\
12.53\end{array}$ & $\begin{array}{l}50.65 \pm \\
11.79\end{array}$ & $498 / 463$ & $M R I$ & $\begin{array}{l}\text { Patients without } \\
\text { LDD }\end{array}$ & rs1800796 & 0.30 & 15 \\
\hline
\end{tabular}


Table 2 Distribution of genotypes and alleles of IL-6 and IL-10 gene polymorphisms in LDD patients and controls

\begin{tabular}{|c|c|c|c|c|c|c|c|c|c|c|c|c|c|}
\hline \multicolumn{3}{|l|}{ Study } & \multicolumn{5}{|c|}{ Case group } & \multicolumn{5}{|c|}{ Control group } & \multirow{2}{*}{$\begin{array}{l}\text { HWE } \\
\text { for } \\
\text { control }\end{array}$} \\
\hline Author & Year & Ethnicity & 11 & 12 & 22 & 1 & 2 & 11 & 12 & 22 & 1 & 2 & \\
\hline \multicolumn{14}{|l|}{ rs1800795 (G vs. C) } \\
\hline Noponen-Hietala [10] & 2005 & European & 47 & 77 & 31 & 171 & 139 & 37 & 89 & 53 & 163 & 195 & 0.97 \\
\hline Eskola [11] & 2010 & European & 19 & 33 & 14 & 71 & 61 & 37 & 79 & 38 & 153 & 155 & 0.74 \\
\hline Kelempisioti [13] & 2011 & European & 35 & 77 & 38 & 147 & 153 & 39 & 117 & 90 & 196 & 296 & 0.92 \\
\hline Huang [14] & 2017 & Asian & 264 & 3 & 0 & 531 & 3 & 297 & 3 & 0 & 597 & 3 & 0.93 \\
\hline \multicolumn{14}{|l|}{ rs1800796 (C vs. G) } \\
\hline Noponen-Hietala [10] & 2005 & European & 0 & 5 & 150 & 5 & 305 & 0 & 15 & 164 & 16 & 342 & 0.55 \\
\hline Eskola [11] & 2010 & European & 1 & 10 & 55 & 12 & 120 & 1 & 12 & 141 & 14 & 294 & 0.20 \\
\hline Kelempisioti [13] & 2011 & European & 0 & 9 & 141 & 9 & 291 & 0 & 15 & 231 & 15 & 477 & 0.62 \\
\hline Huang [14] & 2017 & Asian & 138 & 113 & 16 & 389 & 145 & 193 & 102 & 5 & 488 & 112 & 0.03 \\
\hline Zhu [15] & 2017 & Asian & 55 & 233 & 210 & 653 & 343 & 239 & 181 & 43 & 659 & 267 & 0.30 \\
\hline \multicolumn{14}{|l|}{ rs1800797 (G vs. A) } \\
\hline Noponen-Hietala [10] & 2005 & European & 48 & 76 & 31 & 172 & 138 & 37 & 89 & 53 & 163 & 195 & 0.97 \\
\hline Eskola [11] & 2010 & European & 17 & 34 & 15 & 68 & 64 & 37 & 79 & 38 & 153 & 155 & 0.74 \\
\hline Kelempisioti [13] & 2011 & European & 34 & 79 & 37 & 147 & 153 & 43 & 116 & 87 & 201 & 291 & 0.68 \\
\hline \multicolumn{14}{|l|}{ rs13306435 (A vs. T) } \\
\hline Noponen-Hietala [10] & 2005 & European & 0 & 16 & 139 & 16 & 294 & 0 & 4 & 175 & 4 & 354 & 0.87 \\
\hline Eskola [11] & 2010 & European & 0 & 1 & 65 & 1 & 131 & 0 & 4 & 150 & 4 & 304 & 0.87 \\
\hline \multicolumn{14}{|l|}{ rs2069849 (T vs. C) } \\
\hline Noponen-Hietala [10] & 2005 & European & 0 & 8 & 147 & 8 & 302 & 1 & 17 & 161 & 19 & 339 & 0.46 \\
\hline Eskola [11] & 2010 & European & 0 & 3 & 63 & 3 & 129 & 0 & 11 & 143 & 11 & 297 & 0.64 \\
\hline \multicolumn{14}{|l|}{ rs1800871 (A vs. C) } \\
\hline Lin [13] & 2011 & Asian & 150 & 113 & 57 & 413 & 227 & 89 & 119 & 61 & 297 & 241 & 0.08 \\
\hline Huang [14] & 2017 & Asian & 91 & 143 & 33 & 325 & 209 & 119 & 153 & 28 & 391 & 209 & 0.03 \\
\hline \multicolumn{14}{|l|}{ rs1800896 (G vs. A) } \\
\hline Noponen-Hietala [10] & 2005 & European & 32 & 76 & 47 & 140 & 170 & 34 & 88 & 57 & 156 & 202 & 0.99 \\
\hline Lin [13] & 2011 & Asian & 118 & 113 & 89 & 349 & 291 & 122 & 93 & 54 & 337 & 201 & $<0.01$ \\
\hline Huang [14] & 2017 & Asian & 0 & 70 & 197 & 70 & 464 & 0 & 35 & 265 & 35 & 565 & 0.28 \\
\hline
\end{tabular}

11, 12, and 22 indicate GG GC CC for rs 1800795, CC CG GG for rs1800796, GG GA AA for rs1800797, AA AT Tा for rs13306435, Tा TC CC for rs209849, AA AC CC for rs1800871, and GG GA AA for rs1800896 respectively

\section{Immunohistochemistry}

The intervertebral disc tissue was fixed in $10 \%$ neutral buffered formalin within $1 \mathrm{~h}$ after surgical excision and was embedded in paraffin for serial sectioning at $3 \mu \mathrm{m}$. After routinely xylene dewaxing and gradient ethanol hydration, the sections were incubated in $0.01 \mathrm{~mol} / \mathrm{L}$ citrate buffer $(\mathrm{pH}=6)$. The sections were immersed in 3\% hydrogen peroxide for $10 \mathrm{~min}$ and subsequently rinsed three times with PBS buffer solution for $3 \mathrm{~min}$ each time at room temperature. Nonspecific binding was blocked with $3 \%$ normal goat serum in a phosphate-buffered saline solution $(\mathrm{pH}=$ 7.4) for $15 \mathrm{~min}$ at room temperature. Primary antibody (polyclonal rabbit anti-IL-6/anti-IL-10, ab6672/ ab34843, Abcam, Cambridge, UK) was diluted at 1:
400 and incubated at $4{ }^{\circ} \mathrm{C}$ overnight. After washing, it was followed by secondary antibody (Biotin-labeled Goat Anti-Rabbit IgG, SP-9001, ZYMED, USA) for 15 min at room temperature. Then, the sections were incubated in Horseradish Peroxidase Streptavidin for 15 min. DAB reagent was added to the section that was examined by microscope and incubated for $10 \mathrm{~min}$ at room temperature. Sections were counterstained with hematoxylin for $10 \mathrm{~min}$, dehydrated through several baths of graded hydrochloric alcohol and xylene dehydration, and then mounted using Neutral Balsam. The results of immunohistochemistry (IHC) were obtained using an Olympus BX43 upright microscope (Olympus Optical, Tokyo, Japan). In addition, fresh intervertebral disc tissues were collected to obtain the total 
Table 3 Association test and heterogeneity test of IL-6 and IL-10 gene polymorphisms (rs1800795, rs1800796, rs1800797, rs13306435, rs2069849, rs1800871, and rs1800896)

\begin{tabular}{|c|c|c|c|c|c|c|c|}
\hline \multirow[t]{2}{*}{ SNP } & \multirow[t]{2}{*}{ Genetic model } & \multirow{2}{*}{$\begin{array}{l}\text { Analysis } \\
\text { model }\end{array}$} & \multicolumn{3}{|c|}{ Test of association } & \multicolumn{2}{|c|}{ Heterogeneity test } \\
\hline & & & $\overline{\mathrm{OR}}$ & $95 \% \mathrm{Cl}$ & $P$ value & $\bar{P}$ & $P_{\text {het }}$ \\
\hline \multirow[t]{9}{*}{ rs1800795 } & Allelic & & & & & & \\
\hline & G vs. C & Fixed & 1.39 & {$[1.15,1.67]$} & 0.0005 & $0 \%$ & 0.77 \\
\hline & Codominant model & & & & & & \\
\hline & GG vs.CC & Fixed & 1.96 & {$[1.34,2.86]$} & 0.0005 & $0 \%$ & 0.66 \\
\hline & GC vs. CC & Fixed & 1.44 & {$[1.04,1.98]$} & 0.03 & $0 \%$ & 0.77 \\
\hline & Dominant model & & & & & & \\
\hline & $\mathrm{CC}+\mathrm{GC}$ vs. GG & Fixed & 0.66 & {$[0.48,0.89]$} & 0.007 & $0 \%$ & 0.83 \\
\hline & Recessive model & & & & & & \\
\hline & $\mathrm{GG}+\mathrm{GC}$ vs. CC & Fixed & 1.59 & {$[1.17,2.15]$} & 0.003 & $0 \%$ & 0.70 \\
\hline \multirow[t]{9}{*}{ rs1800796 } & Allelic & & & & & & \\
\hline & C vs. G & Random & 0.78 & {$[0.55,1.10]$} & 0.16 & $63 \%$ & 0.03 \\
\hline & Codominant model & & & & & & \\
\hline & CC vs. GG & Random & 0.19 & {$[0.03,1.06]$} & 0.06 & $86 \%$ & 0.0008 \\
\hline & CG vs. GG & Random & 0.58 & {$[0.25,1.32]$} & 0.19 & $82 \%$ & 0.0002 \\
\hline & Dominant model & & & & & & \\
\hline & $\mathrm{GG}+\mathrm{CG}$ vs. CC & Random & 2.64 & {$[0.61,11.40]$} & 0.19 & $96 \%$ & $<0.00001$ \\
\hline & Recessive model & & & & & & \\
\hline & $\mathrm{CC}+\mathrm{CG}$ vs. GG & Random & 0.46 & {$[0.15,1.42]$} & 0.18 & $91 \%$ & $<0.00001$ \\
\hline \multirow[t]{9}{*}{ rs1800797 } & Allelic & & & & & & \\
\hline & G vs. A & Fixed & 1.35 & {$[1.12,1.63]$} & 0.002 & $0 \%$ & 0.44 \\
\hline & Codominant model & & & & & & \\
\hline & GG vs. AA & Fixed & 1.80 & {$[1.23,2.63]$} & 0.002 & $0 \%$ & 0.47 \\
\hline & GA vs. AA & Fixed & 1.44 & {$[1.04,1.98]$} & 0.03 & $0 \%$ & 0.68 \\
\hline & Dominant model & & & & & & \\
\hline & $\mathrm{AA}+\mathrm{GA}$ vs. GG & Fixed & 0.70 & {$[0.51,0.95]$} & 0.02 & $0 \%$ & 0.56 \\
\hline & Recessive model & & & & & & \\
\hline & $\mathrm{GG}+\mathrm{GA}$ vs. $A A$ & Fixed & 1.55 & {$[1.14,2.10]$} & 0.005 & $0 \%$ & 0.58 \\
\hline \multirow[t]{9}{*}{ rs13306435 } & Allelic & & & & & & \\
\hline & A vs. T & Random & 2.09 & {$[0.27,15.87]$} & 0.48 & $65 \%$ & 0.09 \\
\hline & Codominant model & & & & & & \\
\hline & AA vs. TT & Fixed & NM & NM & NM & NM & NM \\
\hline & AT vs. TT & Random & 2.12 & {$[0.26,16.97]$} & 0.48 & $66 \%$ & 0.09 \\
\hline & Dominant model & & & & & & \\
\hline & TT+AT vs. AA & Fixed & NM & NM & NM & NM & NM \\
\hline & Recessive model & & & & & & \\
\hline & AA+AT vs. TT & Random & 2.12 & {$[0.26,16.97]$} & 0.48 & $66 \%$ & 0.09 \\
\hline \multirow[t]{5}{*}{ rs2069849 } & Allelic & & & & & & \\
\hline & T vs. C & Fixed & 0.52 & {$[0.25,1.04]$} & 0.07 & $0 \%$ & 0.72 \\
\hline & Codominant model & & & & & & \\
\hline & TT vs. CC & Fixed & 0.36 & {$[0.01,9.03]$} & 0.54 & NM & NM \\
\hline & TC vs. CC & Fixed & 0.55 & {$[0.26,1.13]$} & 0.10 & $0 \%$ & 0.82 \\
\hline
\end{tabular}

Dominant model 
Table 3 Association test and heterogeneity test of IL-6 and IL-10 gene polymorphisms (rs1800795, rs1800796, rs1800797, rs13306435, rs2069849, rs1800871, and rs1800896) (Continued)

\begin{tabular}{|c|c|c|c|c|c|c|c|}
\hline \multirow[t]{2}{*}{ SNP } & \multirow[t]{2}{*}{ Genetic model } & \multirow{2}{*}{$\begin{array}{l}\text { Analysis } \\
\text { model }\end{array}$} & \multicolumn{3}{|c|}{ Test of association } & \multicolumn{2}{|c|}{ Heterogeneity test } \\
\hline & & & OR & $95 \% \mathrm{Cl}$ & $P$ value & 2 & $P_{\text {het }}$ \\
\hline & CC+TC vs. TT & Fixed & 2.61 & {$[0.11,64.62]$} & 0.56 & NM & NM \\
\hline & Recessive model & & & & & & \\
\hline & TT+TC vs. CC & Fixed & 0.52 & {$[0.25,1.08]$} & 0.08 & $0 \%$ & 0.76 \\
\hline \multirow[t]{9}{*}{ rs1800871 } & Allelic & & & & & & \\
\hline & A vs. C & Random & 1.11 & {$[0.63,1.95]$} & 0.72 & $91 \%$ & 0.0008 \\
\hline & Codominant model & & & & & & \\
\hline & AA vs. CC & Random & 1.10 & {$[0.40,2.99]$} & 0.85 & $87 \%$ & 0.006 \\
\hline & AC vs. CC & Fixed & 0.92 & {$[0.65,1.30]$} & 0.65 & $0 \%$ & 0.49 \\
\hline & Dominant model & & & & & & \\
\hline & $C C+A C$ vs. $A A$ & Random & 0.84 & {$[0.38,1.88]$} & 0.68 & $91 \%$ & 0.0008 \\
\hline & Recessive model & & & & & & \\
\hline & $A A+A C$ vs. $C C$ & Random & 1.02 & {$[0.56,1.86]$} & 0.95 & $69 \%$ & 0.07 \\
\hline \multirow[t]{9}{*}{ rs1800896 } & Allelic & & & & & & \\
\hline & G vs. A & Random & 1.2 & {$[0.64,2.88]$} & 0.57 & $92 \%$ & $<0.00001$ \\
\hline & Codominant model & & & & & & \\
\hline & GG vs. AA & Random & 0.79 & {$[0.41,1.50]$} & 0.47 & $67 \%$ & 0.08 \\
\hline & GA vs. AA & Random & 1.28 & {$[0.58,2.97]$} & 0.54 & $89 \%$ & 0.0002 \\
\hline & Dominant model & & & & & & \\
\hline & $A A+G A$ vs. GG & Fixed & 1.25 & {$[0.95,1.66]$} & 0.11 & $50 \%$ & 0.16 \\
\hline & Recessive model & & & & & & \\
\hline & $\mathrm{GG}+\mathrm{GA}$ vs. $\mathrm{AA}$ & Random & 1.23 & {$[0.53,2.83]$} & 0.63 & $91 \%$ & $<0.0001$ \\
\hline
\end{tabular}

NM not mentioned, SNP single nucleotide polymorphisms, $O R$ indicates odds ratio, $C l$ indicates confidence interval, $P_{\text {het }}$ indicates $P$ value for heterogeneity

RNA. The total mRNA was extracted with TRIzol (Invitrogen Life Technologies, CA, USA). One microgram of the total RNA was used to synthesize complementary DNA (cDNA) using an iScript cDNA Synthesis kit (Quanta Biosciences, MD, USA). Subsequently, real-time PCR amplification was performed using specific primers of target genes and a SYBR Green real-time PCR kit (Quanta Biosciences, MD,
USA). The primer sequences were selected according to previous study [24].

\section{Statistical analysis}

Odds ratio (OR) and 95\% confident intervals (CI) were used to assess the strength of associations between IL-6 and IL-10 gene polymorphisms with LDD susceptibility which were conducted under five genetic models. Chi-

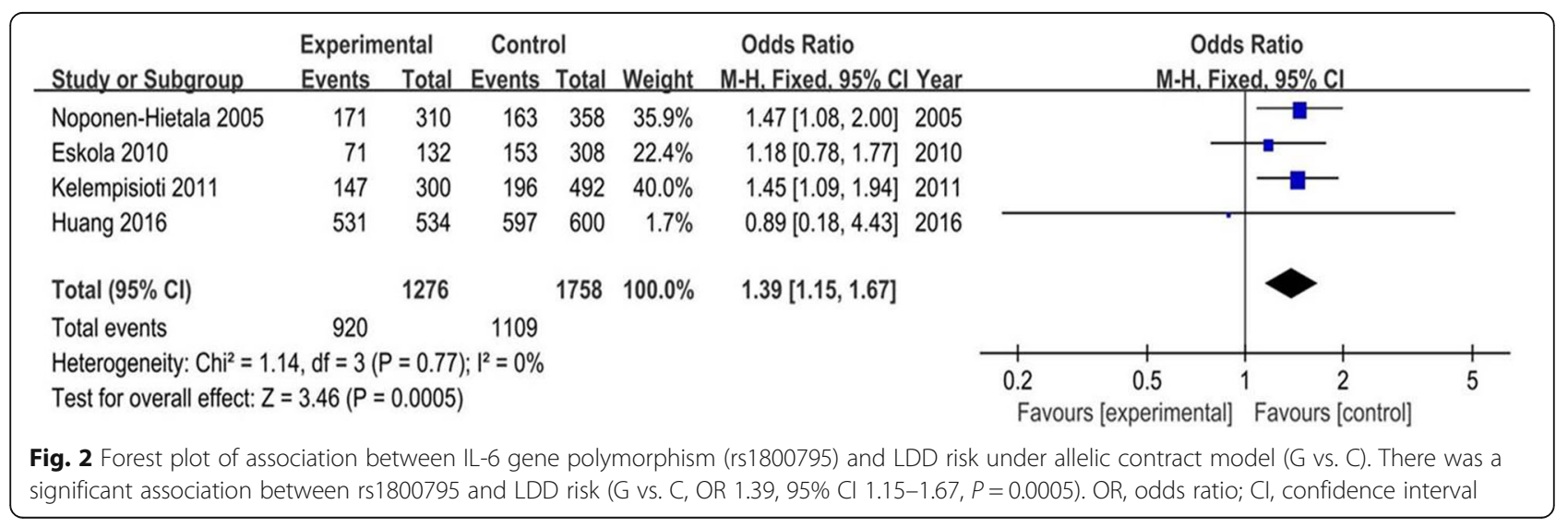




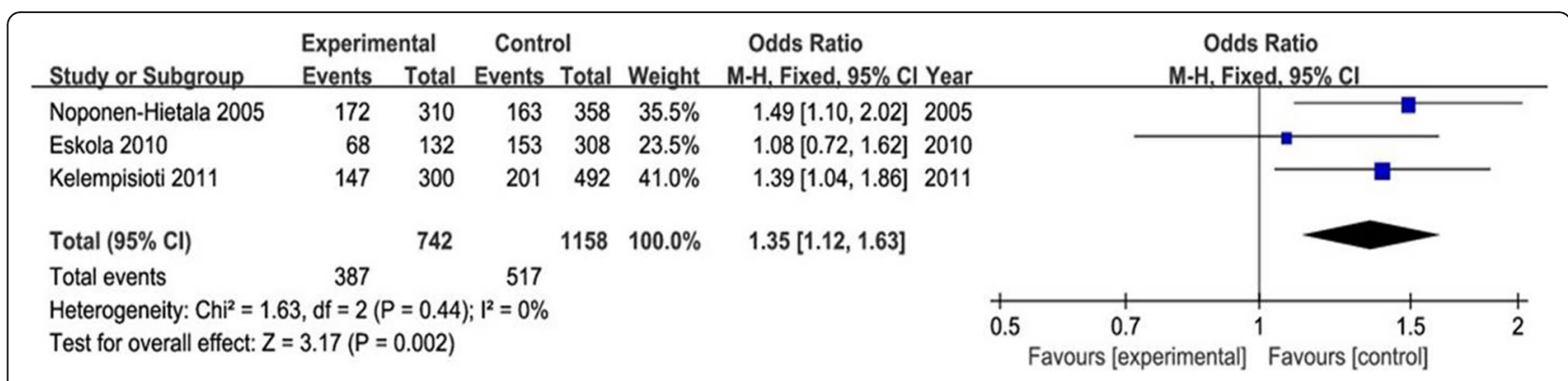

Fig. 3 Forest plot of association between IL-6 gene polymorphism (rs1800797) and LDD risk under allelic contract model (G vs. A). There was a significant association between rs1800797 and LDD risk ( $\mathrm{G}$ vs. $\mathrm{A}, \mathrm{OR} 1.35,95 \% \mathrm{Cl} 1.12-1.63, P=0.002$ ). OR, odds ratio; $\mathrm{Cl}$, confidence interval

square-based $Q$ test and $I^{2}$ test were used to check the heterogeneity among the included studies. A fixed-effect model was used while no heterogeneity existed $(P>0.10$, $\left.I^{2}<50 \%\right)$. Otherwise, a random-effects model was used $\left(P<0.10, I^{2}>50 \%\right)$. For comparisons which have significant heterogeneity, we have performed a sensitive analysis to evaluate the effect of one study on the pooled OR. The Hardy-Weinberg equilibrium of controls was calculated using the HWE Version 1.20 program. Potential publication bias was assessed using Begg's funnel plot and Egger's tests. IHC and RT-PCR data were presented as the mean \pm standard error. Statistical difference between the two groups was evaluated using unpaired Student's $t$ test. Statistical significance was set at $P<$ 0.05. Data analysis was conducted using SPSS 20.0 statistical software (SPSS Inc., Chicago, IL, USA) and Review Manager 5.31 (Nordic Cochrane Center: http://ims. cochrane.org/revman/ download).

\section{Results}

\section{Characteristics of included studies}

The flow chart for the identification of the studies is presented in Fig. 1. A total of 6 studies were finally identified, including 1456 cases and 1611 controls (rs1800795: 4 studies, 638 cases, and 879 controls; rs1800796: 5 studies, 1136 cases, and 1342 controls; rs1800797: 3 studies, 371 cases, and 579 controls; rs2069849: 2 studies, 221 cases, and 333 controls; rs13306435: 2 studies, 221 cases, and 333 controls; rs1800896: 3 studies, 742 cases, and 748 controls; rs1800871: 2 studies, 587 cases, and 569 controls). The main characteristics of included studies are presented in Table 1 and Table 2. For quality assessment, all selected studies are categorized as grade A according to scores ranging from 15 to 19 (Table 1).

\section{IL-6 and IL-10 gene polymorphisms and LDD susceptibility}

The meta-analysis of IL- 6 and IL-10 gene polymorphisms is presented in Table 3. There was a significant association between IL-6 polymorphisms (rs1800795 and rs1800797) and LDD predisposition in five genetic models ( $G$ vs. C, OR 1.39, 95\% CI 1.15-1.67, $P=0.0005$; G vs. A, OR 1.35, 95\% CI 1.12-1.63, $P=0.002$ ) (Figs. 2 and 3). For subgroup analysis, IL-6 rs1800795 polymorphism was negatively associated with LDD risk in the European population in all genetic models except the dominant model $(\mathrm{CC}+\mathrm{CG}$ vs. GG). In addition, no significant association of other IL-6 and IL-10 gene polymorphisms (rs1800796, rs13306435, rs2069849, rs1800871, and rs1800896) with LDD was observed (all $P>0.05$ ).

Sensitivity analysis was performed by excluding one study at a time to analyze the heterogeneity. For rs1800896, the overall results were not altered in all genetic models after omitting the study by Huang et al. [14],

Table 4 Heterogeneity analysis and Egger regression analysis IL-6 and IL-10 gene polymorphisms (rs1800795, rs1800796, rs1800797, rs13306435, rs2069849, rs1800871, and rs1800896)

\begin{tabular}{|c|c|c|c|c|c|c|c|}
\hline \multirow[t]{2}{*}{ SNP } & \multirow{2}{*}{$\begin{array}{l}\text { Analysis } \\
\text { model }\end{array}$} & \multicolumn{3}{|c|}{ Heterogeneity analysis } & \multicolumn{3}{|c|}{ Egger regression analysis } \\
\hline & & $x^{2}$ & $P$ & $P^{2}(\%)$ & $t$ & $95 \% \mathrm{Cl}$ & $P$ \\
\hline rs1800795 & Fixed & 1.14 & 0.77 & 0 & -1.54 & {$[-3.58,1.69]$} & 0.263 \\
\hline rs1800796 & Random & 10.89 & 0.03 & 63 & 0.37 & {$[-4.15,5.24]$} & 0.736 \\
\hline rs1800797 & Fixed & 1.63 & 0.44 & 0 & -4.19 & {$[-14.66,7.38]$} & 0.149 \\
\hline rs13306435 & Random & 2.84 & 0.09 & 65 & & & \\
\hline rs2069849 & Fixed & 0.13 & 0.72 & 0 & & & \\
\hline rs1800871 & Random & 11.17 & 0.0008 & 91 & & & \\
\hline rs1800896 & Random & 24.97 & $<0.00001$ & 92 & 14.47 & {$[1.52,23.42]$} & 0.044 \\
\hline
\end{tabular}


Table $\mathbf{5}$ The result of sensitivity analysis with each study omitted for rs1800796 in IL-6 gene and rs1800896 in IL-10 gene

\begin{tabular}{llll}
\hline Study omitted & OR & $95 \% \mathrm{Cl}$ & $P$ \\
\hline rs1800796 C/G & & & \\
Noponen-Hietala [10] & 0.84 & {$[0.59,1.19]$} & 0.32 \\
Eskola [11] & 0.70 & {$[0.56,0.87]$} & 0.001 \\
Kelempisioti [12] & 0.76 & {$[0.52,1.12]$} & 0.17 \\
Huang [14] & 0.89 & {$[0.51,1.55]$} & 0.67 \\
Zhu [15] & 0.82 & {$[0.43,1.56]$} & 0.55 \\
rs1800896 G/A & & & \\
Noponen-Hietala [10] & 1.30 & {$[0.39,4.33]$} & 0.67 \\
Lin [13] & 1.59 & {$[0.71,3.57]$} & 0.26 \\
$\quad$ Huang [14] & 0.86 & {$[0.58,1.27]$} & 0.46 \\
\hline OR indicates odds ratio, CI confidence interval & &
\end{tabular}

and the heterogeneity was obviously reduced after omitting Huang's study (Table 4). For rs1800796, the result of the allelic contrast model suggested a negative association between rs1800796 and LDD after excluding the study reported by Eskola et al. [11], and the heterogeneity was significantly reduced after excluding Eskola's study (Table 5). According to Begg's funnel plot and Egger's regression tests, the result indicated no significant publication bias under all genetic models (all $P>$ 0.05 for all models tested) (Fig. 4).

\section{IL- 6 and IL-10 expressions and LDD}

The proteins of IL- 6 and IL-10 expressed in the cytoplasm were detected in the nucleus pulpous of the intervertebral disc. Compared with the control group, the LDD group had significantly higher expression of IL-6 (IL-6 immunopositive cells, $73.68 \pm 10.99 \%$ vs. $37.23 \pm 6.42 \% ; P<0.001)$. However, there were no significant differences in IL-10 expression between the two groups (IL-10 immunopositive cells, 41.18 \pm $23.56 \%$ vs. $32.79 \pm 20.56 \% ; P=0.325)$. For $R T-P C R$, there were higher IL-6 mRNA levels in the LDD group than those in the control group $(P<0.001)$. No significant differences in IL-10 expression levels were found in the two groups $(P=0.112)$ (Fig. 5).

\section{Discussion}

Rigal and colleagues [25] initially used a meta-analysis to validate the effects of genetic polymorphisms on disc degeneration and demonstrated that IL-6 rs1800797 polymorphism was identified as a positive gene locus of LDD. Recently, several studies were published to explicate the association between IL- 6 and IL-10 gene polymorphisms and LDD risk [14, 15]. Larger sample size of meta-analysis was essential to examine reliability and accuracy of the conclusion [26]. Hence, a comprehensive meta-analysis needs to update with data from the latest studies. The current study, including 1456 cases and

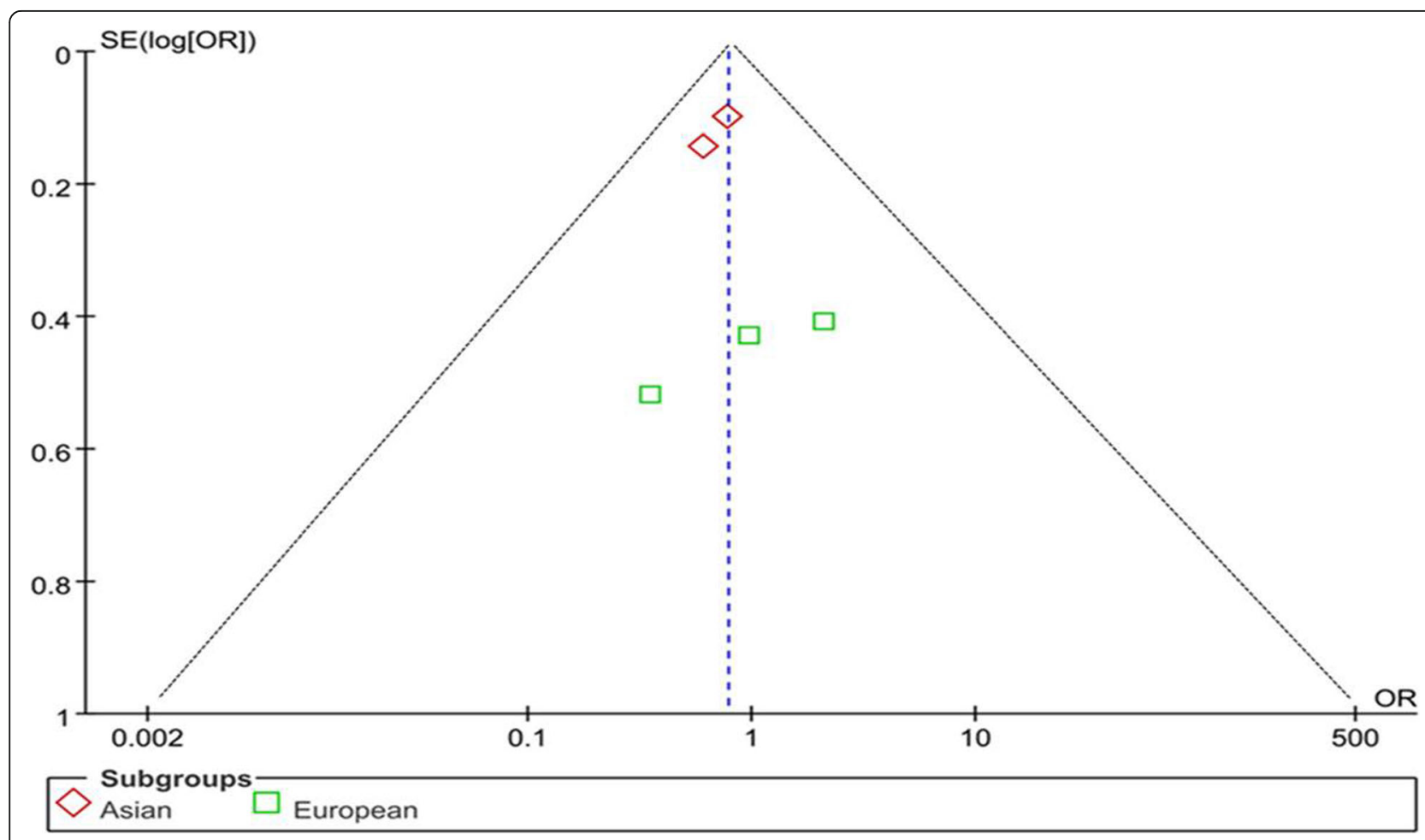

Fig. 4 Funnel plot analysis for publication bias in selection of studies on IL-6 gene polymorphism (rs1800796) under allelic contract model (C vs. $\mathrm{G})$. There was no significant publication bias $(P>0.05)$ 


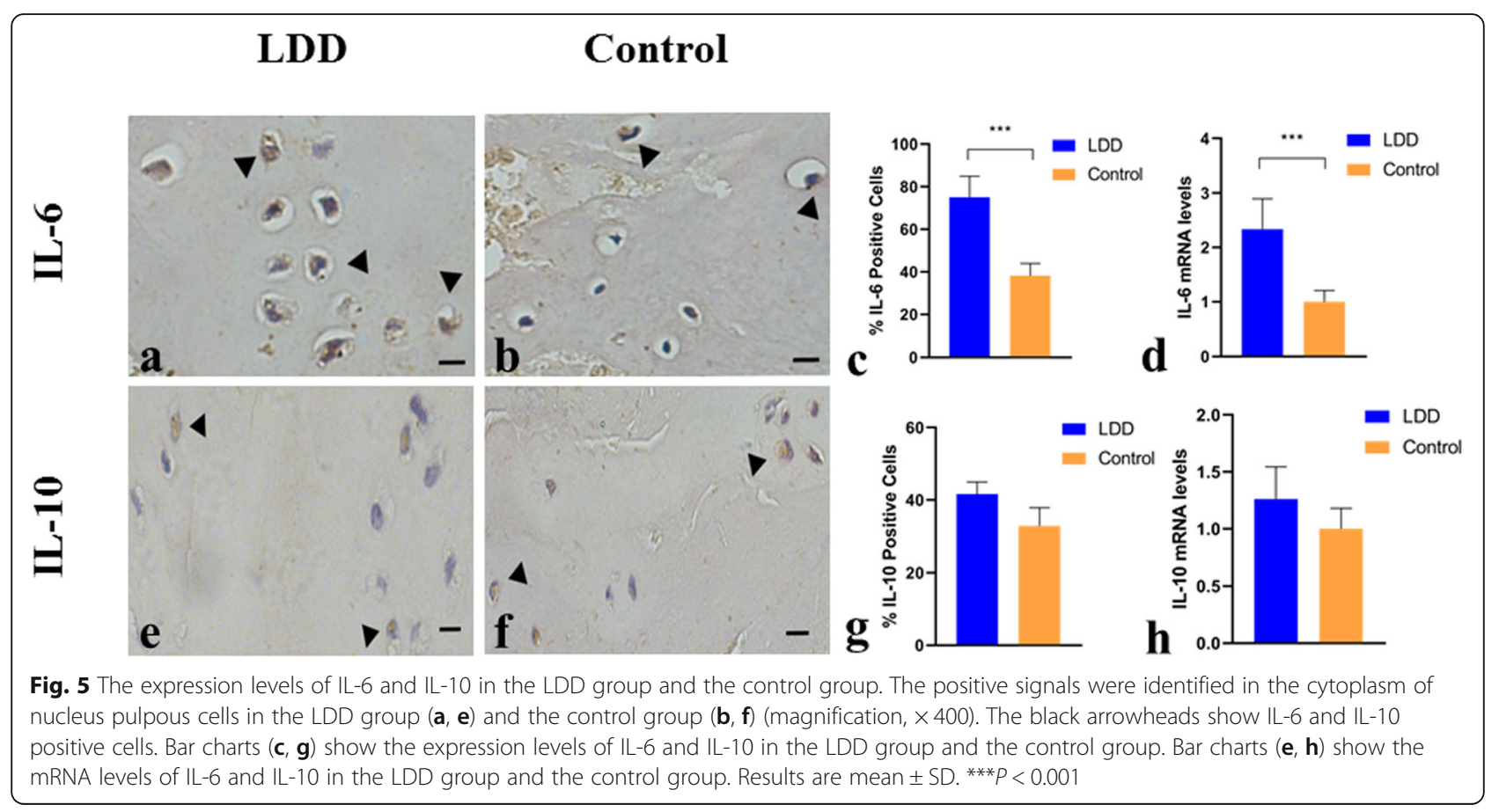

1611 controls, showed that two IL-6 gene polymorphisms (rs1800795 and rs1800797) were significantly associated with susceptibility to LDD in all genetic models. In accordance with Northern European reports [11, 12], our study identified that the $G$ allele represented approximately 1.38 - and 1.35 -fold increased risk factor for developing disc degeneration. Furthermore, we performed the subgroup analysis stratified by ethnicity. After subgroup analysis, it revealed that the IL-6 rs1800795 polymorphism is associated with LDD in European population but not in Asian population. The heterogeneity of our study should be observed in interpreting the result. Various study designs and genetic background may be explicated the main causes of heterogeneity. More importantly, the presence of heterogeneity may result from the different diagnostic criteria of LDD and phenotype selection [27, 28]. There are obvious discrepancies in the clinical phenotype of LDD patients, such as annular tears, disc herniation, spinal stenosis, and spondylolisthesis. We could postulate that various phenotypes might represent different disease courses of LDD, which may be influenced by variable genetic polymorphisms [29].

IL-6 is a pro-inflammatory cytokine which plays a vital role in the regulation of host immune response in intervertebral disc [30]. We performed an in silico analysis for evaluating the possible functional implication of rs1800795 and rs1800797 polymorphism by using rSNPBase (http:// rsnp3.psych.ac.cn/) [31] and found that these two SNPs were located within the promoter of IL-6 gene, which had a possible transcriptional regulatory effect. The $\mathrm{G}$ to $\mathrm{C}$ polymorphism at position-174 of the IL-6 gene (rs1800795) causes differential activity in promoter constructs which upregulates IL-6 gene transcription [32]. The $\mathrm{G}$ allele of rs 1800795 promotes higher circulating levels of IL-6 in patients with sepsis [33]. However, the relationship between IL-6 polymorphisms and IL-6 expression in LDD patients has not been reported. Our metaanalysis confirmed that IL-6 gene polymorphisms were significantly associated with LDD, and the G allele of rs1800795 and rs1800797 represented the risk factors for LDD. Furthermore, the results of IHC and RT-PCR analysis showed that increased IL-6 expression levels were found in the degenerated disc. Compared with GC/CC or GA/AA genotypes, GG genotypes of rs1800795 and rs1800797 were associated with higher levels of IL-6 expression. Thus, we postulated that rs 1800795 GG or/and rs1800797 GG genotypes were the genetic risk factors for progression of LDD, probably by decreasing the expression of IL-6. IL-6 rs1800795 and rs1800797 polymorphisms, located within regulatory regions, could be part of RNAbinding protein site and could be involved in RNAbinding which is protein-mediated. Previous studies showed that IL-6 significantly induced disc degeneration by activating STAT3 and $\beta$-catenin signaling pathways $[30$, 34]. It seems to indicate that IL-6 might act synergistically with other genetic factors contributing to the risk of disc degeneration.

Rs13306435 and rs2069849 were located in exon 5 of IL-6 gene. The T>A variation of rs13306435 changed an amino acid from Asp to Glu. The SNP rs2069849 $(\mathrm{C}>\mathrm{T})$ was a synonymous variant. The $\mathrm{T}$ 
allele of 13306435 and the C allele of rs2069849 had been reported previously to be associated with increased expression and plasma levels of IL-6 [32]. However, other studies did not found any association [35, 36]. Karppinen et al. [37] supposed that these two SNPs were unlikely to cause disease but may be in linkage disequilibrium with the functional mutation some distance away from it. In addition, rs1800871 and rs1800896 were two promoter polymorphisms of IL-10, which may lead to alteration of the specific transcription factor recognition sites [38]. Gibson et al. found that two SNPs were not associated with the differential IL-10 expression in LDD, and other polymorphisms, particularly those in the distal part of the promoter, may have an effect on IL-10 production [39]. Based on recent research, the effects of IL-10 promoter polymorphisms on gene expression are likely to be more complex than what has been initially expected [40].

Several limitations of this study should be acknowledged. First, heterogeneity could result from different phenotype selection and diagnostic criteria of LDD. It may exert an influence on the reliability of metaanalysis. Second, we did not perform the subgroup analysis stratified by gender, age, and environmental factors as the original data were unavailable. Third, for IHC and RT-PCR studies, the sample size was relatively small, which may increase the risk of false positive. More evidence is needed to validate these associations.

\section{Conclusions}

Our study indicated that IL-6 gene polymorphisms (rs1800795 and rs1800797) were significantly associated with susceptibility to LDD. IL-6 expression levels may be may be an important risk factor for LDD.

\section{Abbreviations}

IL-10: Interleukin-10; IL-6: Interleukin-6; LBP: Low back pain; LDD: Lumbar disc degeneration; SNP: Single nucleotide polymorphism

\section{Acknowledgements}

The authors thank all the participants in this study.

\begin{abstract}
Authors' contributions
$\mathrm{HJ}$ conceived and designed the study. SW and HW were involved in the data search and selection of data, analyzed the data, and wrote the manuscript. YG was involved in the data search and selection of data and analyzed the data. JW and DM were involved in the data search and analyzed the data. SW and YG were involved in the data search and selection of data. QJW analyzed the data and contributed the analysis tools. YG was involved in the IHC study. All authors read and approved the final manuscript.
\end{abstract}

\section{Funding}

This work was supported by the Natural Science Foundation of China (81860406/ 81460353/81560371), Guangxi Natural Science Foundation (2018GXNSFAA281127/ 2015GXNSFBA139167), and Medical Excellence Award Funded by the Creative Research Development Grant from the First Affiliated Hospital of Guangxi Medical University.

\section{Availability of data and materials}

Please contact the authors for data requests.

\section{Ethics approval and consent to participate}

The histology study was approved by the Ethics Committee of the First Affiliated Hospital of Guangxi Medical University. All the experimental protocol and the methods were carried out in accordance with the relevant guidelines and regulations and complied with the principles of the Declaration of Helsinki. Written informed consent was achieved from each participant.

\section{Consent for publication}

Not applicable.

\section{Competing interests}

The authors declare that they have no competing interests.

Received: 22 September 2019 Accepted: 11 February 2020 Published online: 18 February 2020

\section{References}

1. Patrick N, Emanski E, Knaub MA. Acute and chronic low back pain. Med Clin North Am. 2014;98:777-89 xii.

2. Selkirk SM, Ruff R. Low back pain, radiculopathy. Handb Clin Neurol. 2016; 136:1027-33.

3. Yang S, Kim W, Choi KH, Yi YG. Influence of occupation on lumbar spine degeneration in men: the Korean national health and Nutrition Examination Survey 2010-2013. Int Arch Occup Environ Health. 2016;89:1321-8.

4. Zheng CJ, Chen J. Disc degeneration implies low back pain. Theor Biol Med Model. 2015:12:24

5. Zawilla NH, Darweesh H, Mansour N, Helal S, Taha FM, Awadallah M, El Shazly R. Matrix metalloproteinase-3, vitamin D receptor gene polymorphisms, and occupational risk factors in lumbar disc degeneration. J Occup Rehabil. 2014;24:370-81

6. Teraguchi M, Yoshimura N, Hashizume H, Muraki S, Yamada H, Minamide A Oka H, Ishimoto $Y$, Nagata K, Kagotani R, et al. Prevalence and distribution of intervertebral disc degeneration over the entire spine in a populationbased cohort: the Wakayama Spine Study. Osteoarthr Cartil. 2014;22:104-10.

7. Wang S, Wang L, Wang Y, Du C, Zhang M, Fan Y. Biomechanical analysis of combining head-down tilt traction with vibration for different grades of degeneration of the lumbar spine. Med Eng Phys. 2017;39:83-93.

8. Kalichman L, Hunter DJ. The genetics of intervertebral disc degeneration. Associated genes. Joint Bone Spine. 2008;75:388-96.

9. Basaran R, Senol M, Ozkanli S, Efendioglu M, Kaner T. Correlation of matrix metalloproteinase (MMP)-1, -2, -3 , and -9 expressions with demographic and radiological features in primary lumbar intervertebral disc disease. J Clin Neurosci. 2017:41:46-9.

10. Noponen-Hietala N, Virtanen I, Karttunen R, Schwenke S, Jakkula E, Li H, Merikivi R, Barral S, Ott J, Karppinen J, Ala-Kokko L. Genetic variations in IL6 associate with intervertebral disc disease characterized by sciatica. Pain. 2005;114:186-94.

11. Eskola PJ, Kjaer P, Daavittila IM, Solovieva S, Okuloff A, Sorensen JS, Wedderkopp N, Ala-Kokko L, Mannikko M, Karppinen Jl. Genetic risk factors of disc degeneration among 12-14-year-old Danish children: a population study. Int J Mol Epidemiol Genet. 2010;1:158-65.

12. Kelempisioti A, Eskola PJ, Okuloff A, Karjalainen U, Takatalo J, Daavittila I, Niinimaki J, Sequeiros RB, Tervonen O, Solovieva S, et al. Genetic susceptibility of intervertebral disc degeneration among young Finnish adults. BMC Med Genet. 2011:12:153.

13. Lin WP, Lin JH, Chen XW, Wu CY, Zhang LQ, Huang ZD, Lai JM. Interleukin10 promoter polymorphisms associated with susceptibility to lumbar disc degeneration in a Chinese cohort. Genet Mol Res. 2011;10:1719-27.

14. Huang X, Chen F, Zhao J, Wang D, Jing S, Li H, Meng C. Interleukin 6 (IL-6) and IL-10 promoter region polymorphisms are associated with risk of lumbar disc herniation in a Northern Chinese Han population. Genet Test Mol Biomarkers. 2017:21:17-23.

15. Zhu Y, Li S, Niu F, Yan M, Li J, Jia H, Yang X. Association between IL4, IL6 gene polymorphism and lumbar disc degeneration in Chinese population. Oncotarget. 2017:8:89064-71

16. Saraiva M, O'Garra A. The regulation of $\mathrm{IL}-10$ production by immune cells. Nat Rev Immunol. 2010;10:170-81. 
17. Risbud MV, Shapiro IM. Role of cytokines in intervertebral disc degeneration: pain and disc content. Nat Rev Rheumatol. 2014;10:44-56.

18. Bennermo M, Held C, Stemme S, Ericsson CG, Silveira A, Green F, Tornvall P. Genetic predisposition of the interleukin- 6 response to inflammation: implications for a variety of major diseases? Clin Chem. 2004;50:2136-40.

19. Feitosa RN, Vallinoto AC, Vasconcelos PF, Azevedo Rdo S, Azevedo VN, Machado LF, Lima SS, Ishak Mde O, Ishak R. Gene polymorphisms and serum levels of pro- and anti-inflammatory markers in dengue viral infections. Viral Immunol. 2016;29:379-88.

20. Moher D, Shamseer L, Clarke M, Ghersi D, Liberati A, Petticrew M, Shekelle P, Stewart LA, Group P-P. Preferred reporting items for systematic review and meta-analysis protocols (PRISMA-P) 2015 statement. Syst Rev. 2015;4:1.

21. National CASP. Collaboration for qualitative methodologies: 10 questions to help you make sense of qualitative research. Milton Keynes: Milton Keynes Primary Care Trust; 2006.

22. Yang Q, Liu Y, Guan Y, Zhan X, Xiao Z, Jiang H, Wei Q. Vitamin D receptor gene polymorphisms and plasma levels are associated with lumbar disc degeneration. Sci Rep. 2019;9:7829.

23. Schneiderman G, Flannigan B, Kingston S, Thomas J, Dillin WH, Watkins RG. Magnetic resonance imaging in the diagnosis of disc degeneration: correlation with discography. Spine (Phila Pa 1976). 1987;12:276-81.

24. Maher IE, Griffith JE, Lau Q, Reeves T, Higgins DP. Expression profiles of the immune genes CD4, CD8beta, IFNgamma, IL-4, IL-6 and IL-10 in mitogenstimulated koala lymphocytes (Phascolarctos cinereus) by qRT-PCR. PeerJ. 2014;2:e280.

25. Rigal J, Leglise A, Barnetche T, Cogniet A, Aunoble S, Le Huec JC. Metaanalysis of the effects of genetic polymorphisms on intervertebral disc degeneration. Eur Spine J. 2017;26:2045-52.

26. Furlan AD, Malmivaara A, Chou R, Maher CG, Deyo RA, Schoene M, Bronfort G, van Tulder MW, Editorial Board of the Cochrane Back NG. Updated method guideline for systematic reviews in the Cochrane Back and Neck Group. Spine (Phila Pa 1976). 2015;2015(40):1660-73.

27. Vergroesen PP, Kingma I, Emanuel KS, Hoogendoorn RJ, Welting TJ, van Royen BJ, van Dieen JH, Smit TH. Mechanics and biology in intervertebral disc degeneration: a vicious circle. Osteoarthr Cartil. 2015;23:1057-70.

28. Rajasekaran S, Kanna RM, Senthil N, Raveendran M, Cheung KM, Chan D, Subramaniam S, Shetty AP. Phenotype variations affect genetic association studies of degenerative disc disease: conclusions of analysis of genetic association of 58 single nucleotide polymorphisms with highly specific phenotypes for disc degeneration in 332 subjects. Spine J. 2013;13:1309-20.

29. Jiang H, Yang Q, Jiang J, Zhan X, Xiao Z. Association between COL11A1 (rs1337185) and ADAMTS5 (rs162509) gene polymorphisms and lumbar spine pathologies in Chinese Han population: an observational study. BMJ Open. 2017;7:e015644.

30. Suzuki S, Fujita N, Fujii T, Watanabe K, Yagi M, Tsuji T, Ishii K, Miyamoto T, Horiuchi K, Nakamura M, Matsumoto M. Potential involvement of the IL-6/ JAK/STAT3 pathway in the pathogenesis of intervertebral disc degeneration. Spine (Phila Pa 1976). 2017;42:E817-24.

31. Wen AQ, Wang J, Feng K, Zhu PF, Wang ZG, Jiang JX. Effects of haplotypes in the interleukin 1 beta promoter on lipopolysaccharide-induced interleukin 1 beta expression. Shock. 2006;26:25-30

32. Fishman D, Faulds G, Jeffery R, Mohamed-Ali V, Yudkin JS, Humphries S, Woo $P$. The effect of novel polymorphisms in the interleukin-6 (IL-6) gene on IL-6 transcription and plasma IL-6 levels, and an association with systemic-onset juvenile chronic arthritis. J Clin Invest. 1998;102:1369-76.

33. Jimenez-Sousa MA, Medrano LM, Liu P, Fernandez-Rodriguez A, Almansa R, Gomez-Sanchez E, Ortega A, Heredia-Rodriguez M, Gomez-Pesquera E, Tamayo E, Resino S. IL-6 rs1800795 polymorphism is associated with septic shock-related death in patients who underwent major surgery: a preliminary retrospective study. Ann Intensive Care. 2017;7:22.

34. Chen J, Mei Z, Huang B, Zhang X, Liu J, Shan Z, Wang J, Wang X, Zhao F. IL6/YAP1/beta-catenin signaling is involved in intervertebral disc degeneration. J Cell Physiol. 2019;234:5964-71.

35. Ding L, Shao X, Cao L, Fang W, Yan H, Huang J, Gu A, Yu Z, Qi C, Chang X, $\mathrm{Ni} Z$. Possible role of IL-6 and TIE2 gene polymorphisms in predicting the initial high transport status in patients with peritoneal dialysis: an observational study. BMJ Open. 2016;6:e012967.

36. Farhat SB, de Souza CM, Braosi AP, Kim SH, Tramontina VA, Papalexiou V, Olandoski M, Mira MT, Luczyszyn SM, Trevilatto PC. Complete physical mapping of IL6 reveals a new marker associated with chronic periodontitis. J Periodontal Res. 2017;52:255-61.
37. Karppinen J, Daavittila I, Noponen N, Haapea M, Taimela S, Vanharanta H, Ala-Kokko L, Mannikko M. Is the interleukin-6 haplotype a prognostic factor for sciatica? Eur J Pain. 2008;12:1018-25.

38. Sadler AJ, Williams BR. Interferon-inducible antiviral effectors. Nat Rev Immunol. 2008;8:559-68.

39. Gibson AW, Edberg JC, Wu J, Westendorp RG, Huizinga TW, Kimberly RP. Novel single nucleotide polymorphisms in the distal IL-10 promoter affect IL-10 production and enhance the risk of systemic lupus erythematosus. J Immunol. 2001;166:3915-22.

40. Smith AJ, Humphries SE. Cytokine and cytokine receptor gene polymorphisms and their functionality. Cytokine Growth Factor Rev. 2009; 20:43-59.

\section{Publisher's Note}

Springer Nature remains neutral with regard to jurisdictional claims in published maps and institutional affiliations.
Ready to submit your research? Choose BMC and benefit from:

- fast, convenient online submission

- thorough peer review by experienced researchers in your field

- rapid publication on acceptance

- support for research data, including large and complex data types

- gold Open Access which fosters wider collaboration and increased citations

- maximum visibility for your research: over $100 \mathrm{M}$ website views per year

At BMC, research is always in progress.

Learn more biomedcentral.com/submissions 